Article

\title{
Table Tennis Ball Impacting Racket Polymeric Coatings: Experiments and Modeling of Key Performance Metrics
}

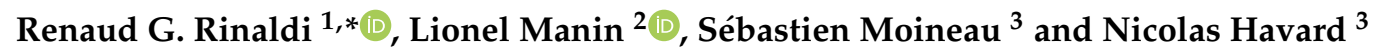 \\ 1 MATEIS, CNRS UMR 5521, INSA-Lyon, Univ Lyon, F-69621 Villeurbanne, France \\ 2 LaMCoS, CNRS UMR 5529, INSA-Lyon, Univ Lyon, F-69621 Villeurbanne, France; lionel.manin@insa-lyon.fr \\ 3 Cornilleau, Bonneuil-Les-Eaux, 60121 Breteuil, France; smoineau@cornilleau.fr (S.M.); \\ nhavard@cornilleau.fr (N.H.) \\ * Correspondence: renaud.rinaldi@insa-lyon.fr; Tel.: +33-04-72-43-62-09
}

Received: 7 November 2018; Accepted: 29 December 2018; Published: 4 January 2019

\begin{abstract}
The performance of a table tennis racket is often associated with subjective or quantitative criteria such as the adhesion, the control and the speed. Overall, the so-called performance aims at characterizing the impact with the ball. Ultimately, the polymeric layers glued onto the wooden blade play a key role, as evidenced in a previous work where the normal linear (no spin) impact of a ball onto polymeric layers was experimentally and numerically investigated. In this work, more realistic loading conditions leading to varying the incident angle and spin of the ball, were explored. While the sole linear restitution coefficient was determined in the anterior normal impact study, new physical metrics were identified to describe fully the trajectory of the reflected ball after impact. A companion 3D finite elements model was developed where the polymeric time-dependent dissipative compliant behavior measured with dynamic mechanical analysis and compression tests was accounted for. The confrontations with the experimental data highlighted the key role of the polymer intrinsic properties along with the friction coefficient between the ball and the polymer external layer.
\end{abstract}

Keywords: impact; polymer; rate dependence; architecture; friction; finite elements

\section{Introduction}

A table tennis racket comprises two polymeric sheets, the foam and the compact that are glued together and on to a wooden paddle. Both polymeric constituents are in their rubbery state at ambient temperature so that they exhibit large and mostly instantaneous reversible deformation when impacted by a ball. Aside from preventing the ball from breaking, these compliant layers trigger the speed and control of the ball and the identification of the design parameters controlling the overall performance is of great importance. Looking at the complex physical phenomenon taking place when the ball impacts a polymeric pad, the friction coefficient, the polymeric intrinsic bulk properties and the layers geometries and architectures correspond to the many potentially adjustable parameters. That being said, when considering the impact problem attention can be focused on the impactor, the target and/or the contact between the two.

While many studies were devoted to studying the kinematic behavior (trajectory and aerodynamics) of a thin shell hollow sphere impacting a flat surface, most of these works used rigid surface, thus being representative of the ball/table impact [1-7]. When the rebound of the ball was the focus of attention, the coefficient of restitution, corresponding to the ratio between the incident and reflected linear velocities could be calculated $[2,8]$. This parameter only relied on the kinematic description of the impact and on global dynamics so that little to no attention was often given to the materials description. In the end, the coefficient of restitution metric characterized the overall speed performance during impact, which corresponded to the performance of a given combination of impactor and target as a whole. 
Impact under normal incidence (and no spin) was often employed to further study the deforming behavior of the ball when in contact with a rigid surface. For both the quasi-static and the dynamic loading regime, the reversible buckling of the thin-wall sphere has been observed and modeled [9-12]. Here, further attention was given to the material mechanical properties. Nevertheless, when used as the model material, the table tennis ball's celluloid constitutive behavior could be described as an elastic perfectly plastic material [11] even though polymers are known to be dissipative rate-dependent materials [13,14]. Recently, Zhang et. al. used an inverse-fitting method to describe the visco-elastic properties of the celluloid material and to model the impact of a table tennis ball impacting a rigid surface, evidencing the need for the account of the polymeric materials rate-dependency [8].

The impact of tennis table ball on a racket with coverings had been thoroughly studied by Tiefenbacher et al. [15] from a macroscopic analysis, both numerically and experimentally. The impact was analyzed based on two restitution coefficients $\left(E_{p a r}\right.$, normal ball velocity, $T_{\text {par }}$ tangential ball velocity). It was shown that the tangential elasticity of the coverings had a high influence on these coefficients, the authors identified a so called 'tangential effect'. The details of the ball—covering contact during impact were not discussed but only analyzed through the previous coefficient of restitution that were determined for numerous coverings and impact conditions (velocity, angle and spin frequency). Following that study, Tiefenbacher et al. [16] had analyzed the influence of special equipment materials on decisive strokes.

The contact conditions during the impact was studied by Cross [17], but for tennis, golf, basketball and baseball balls. The author based his analysis on the measurement of the tangential and normal forces during the ball-surface impact. The tangential force being seen as a parameter that permit identifying the contact condition: sliding, rolling or gripping of the ball on the impacted surface. The author highlighted that depending on its incidence angle and its linear speed and spin frequency, the ball could grip or slide but did not roll on the impacted surface. However, the author did not consider table tennis balls which behave differently from tennis balls, exhibiting for instance a lower mass/volume ratio.

Finite element modelling (FEM) of sports balls impacting rackets, bats, clubs or surfaces has been considered by many authors in the last two decades. Goodwill, Haake and Allen [18-21] extensively investigated the impact of a tennis ball on a rigid surface or on a string bed, studying the contact conditions of the tennis ball with the impacted surface and the reversal of spin. More generally, depending on the sport under investigation, noticeable differences exist between the two impacting bodies. They can be considered rigid, soft, plain or hollow so that the deformations experienced by the ball and/or the target can be large and/or small. Ultimately the corresponding models have to be adapted to these specificities. The constitutive equations, often being nonlinear, has to be implemented in the FEM, requiring most of the time the experimental characterization of the material parameters. Another key point for getting a consistent modeling is the contact formulation and its characteristics: friction coefficient, contact state (stick/slip).

In a recent work, Rinaldi et al. studied the impact response of a ball onto targets corresponding to the polymeric layers of a table tennis paddle [22]. Normal impacts with varying incident linear velocities and no spin were solely considered and joint experimental measurements and 3D FEM were performed. The linear coefficient of restitution corresponded to the quantitative metric chosen for comparison and fair agreement was obtained. The buckling of the ball was confirmed numerically meaning that the sample/ball contact zone corresponded to a ring with its mean radius increasing till the maximum crushing. It is worth noting that the material properties, the geometry and the local contact zone were considered in the model, so that parametric studies allowed to evidence their importance. Nevertheless, the explored impact conditions were away from realistic conditions where angular velocity (ball spin) and non-normal incidence usually prevail.

Thus, the present research aimed at exploring an enhanced set of incident loading conditions, as well as challenging the finite element model in matching the experimental observations. Along with the large set of impact tests, new metrics had been defined, monitored and confronted. 


\section{Materials and Methods}

\subsection{Materials}

The balls and the polymeric pads were provided by the French company Cornilleau@. Section views of the three elements in the pristine state, and reconstructed from micro Computed Tomography $(\mu-\mathrm{CT})$ measurements, are presented in Figure 1, illustrating the geometry, architecture and microstructure of the different constituents.

(a)

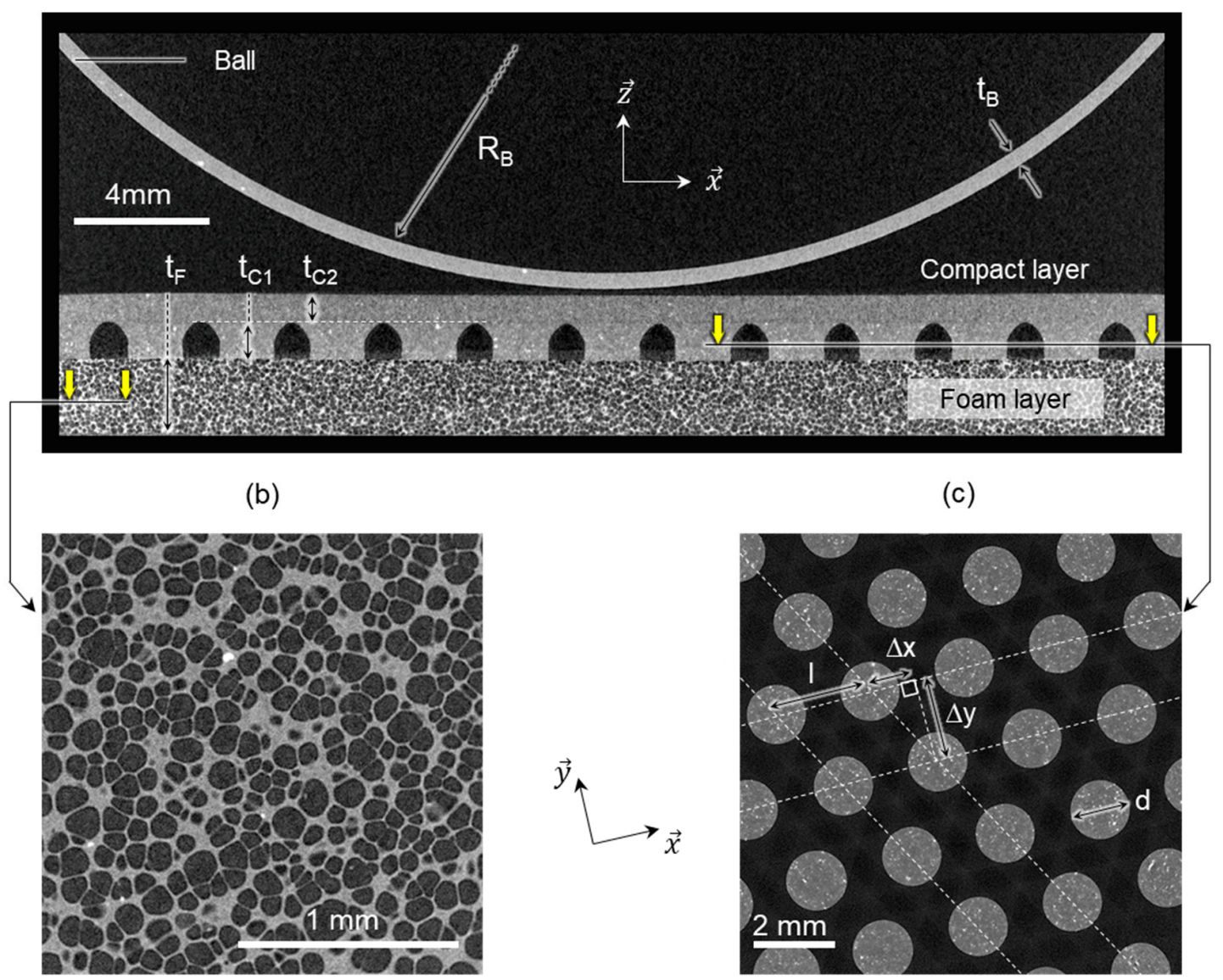

Figure 1. X-ray tomography measurements (a) 2D X-ray tomography imaging of the ball and foam + compact pad (Voxel size $=17 \mu \mathrm{m})$. (b) Section view $(\mathrm{xz})$ of the foam microstructure (Voxel size $=2.5 \mu \mathrm{m}$ ).

(c) Section view (xy) highlighting the periodic pattern of the circular pimples (Voxel size $=17 \mu \mathrm{m}$ ).

Key geometrical parameters and their values are listed in Table 1. They were obtained from the image analysis of the $3 \mathrm{D} \mu-\mathrm{CT}$ data or mentioned otherwise. It is worth noting that the pimples diameter varied throughout the thickness ( $\mathrm{z}$ axis) and that only the mean value was reported.

Table 1. Dimensions of the ball and polymer layers.

\begin{tabular}{lcc}
\hline \multicolumn{1}{c}{ Description } & Symbol & Dimension \\
\hline Ball & $\mathrm{R}_{\mathrm{B}}$ & $20 \mathrm{~mm}$ \\
$\quad$ Radius & $\mathrm{t}_{\mathrm{B}}$ & $0.39 \mathrm{~mm}$ \\
$\quad$ Wall thickness & & \\
\hline Foam & $\mathrm{t}_{\mathrm{F}}$ & $2 \mathrm{~mm}$ \\
$\quad$ Thickness & $\rho_{\mathrm{F}}$ & 0.44 \\
Relative density $^{1}$ & $\rho_{\mathrm{F}}$ & $490 \mathrm{~kg} / \mathrm{m}^{3}$ \\
Density $^{2}$ & \\
\hline
\end{tabular}


Table 1. Cont.

\begin{tabular}{lcc}
\hline \multicolumn{1}{c}{ Description } & Symbol & Dimension \\
\hline Compact & & \\
Thickness of the pimples & $\mathrm{t}_{\mathrm{C} 1}$ & $0.96 \mathrm{~mm}$ \\
Thickness of the dense layer & $\mathrm{t}_{\mathrm{C} 2}$ & $0.81 \mathrm{~mm}$ \\
Spacing & 1 & $2.37 \mathrm{~mm}$ \\
Offset along the x axis & $\triangle \mathrm{x}$ & $2.08 \mathrm{~mm}$ \\
Offset along the y axis & $\triangle \mathrm{y}$ & $1.18 \mathrm{~mm}$ \\
Density of the parent material $^{2}$ & $\rho_{\mathrm{F}}$ & $1000 \mathrm{~kg} / \mathrm{m}^{3}$ \\
\hline
\end{tabular}

${ }^{1}$ determined via thresholding and post-processing the stack of $\mu$-CT images with ImageJ $\odot .{ }^{2}$ determined via measuring and weighting large samples $(100 \mathrm{~m} \times 10 \mathrm{~mm} \times$ total thickness).

\subsection{Methods and Samples Preparation}

A testing apparatus property of Cornilleau $₫$ and illustrated in Figure 2a, had been used to launch table tennis balls horizontally, while controlling the ball's incident linear and spin velocities. The targets consisted of $100 \mathrm{~mm}^{2}$ squares cut from the paddle coverings where the compact and the foam layers were separated if needed. The two diagonals were drawn on the square surface, as shown in Figure $2 b$, in order to visually ascertain the ball impact within the center region of the targets.

The squared samples were taped on to a wooden plate that was screwed on the rigid frame, $260 \mathrm{~mm}$ away from the launcher's end. The target's frame could be rotated around the x-axis so that the incident angle between the ball and the paddle could be controlled. The ball's outside surface was marked with meridians (see Figure 2c): a circular dashed line allowed location of the seal and two solid lines were drawn using a mask. The dashed line was used to visually detect and exclude from the post-processing routine the tests where impact occurred on the seal. Also, the solid lines were used by the in-house post-processing software to dissociate the ball's linear and angular velocities (see Section 2.3).

A camera with long exposure time settings and stroboscopic lights was used to take one image per impact test. The obtained image displayed the target as well as numerous ball prints before and after impact as evidenced in Figure 3a. Consequently, the time $\Delta t$, related to the strobe frequency $f$ $(\Delta t=1 / f)$ elapsed between two consecutive traces. It is worth mentioning that the principle is similar to that used by Carre et al. [23].

(a)

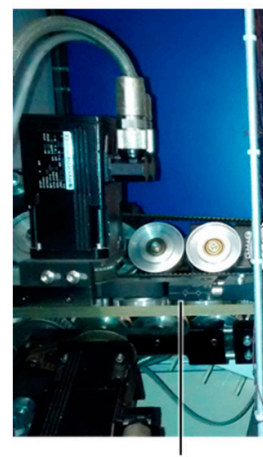

Launcher
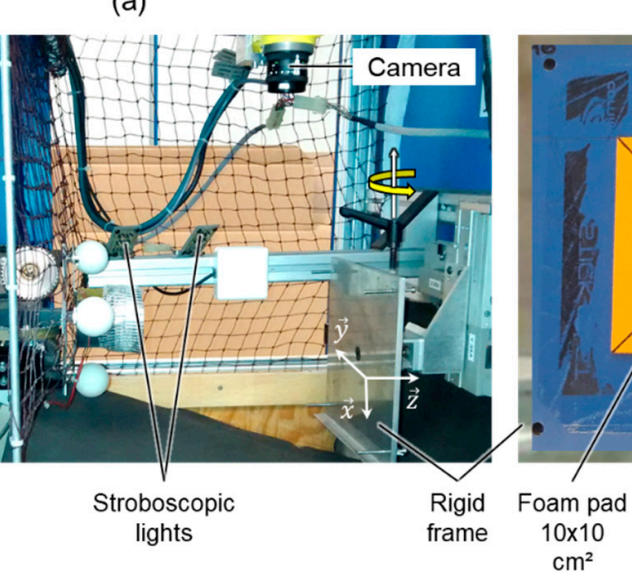

(b)

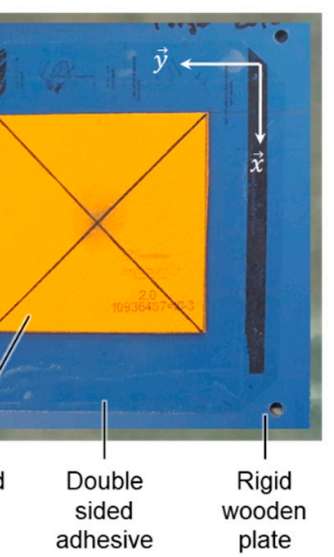

(c)

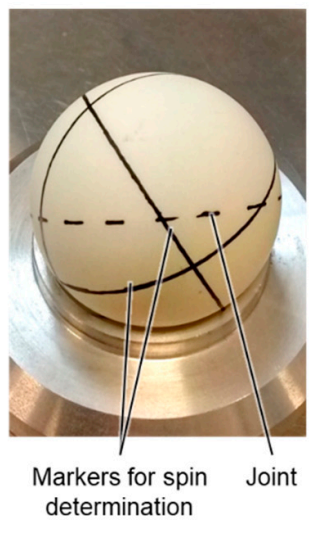

Figure 2. Impact tests. (a) Cornilleau home-made apparatus. (b) Top view of a foam pad glued onto a wooden plate frame. (c) Ball with visual markers drawn.

Ten impacts were performed to characterize a given set of \{Ball-Target - testing conditions\} so that error bars corresponding to the standard deviation were added to the plots displaying the 
experimental results. The measured variabilities apply to both the incident and reflected trajectories and correspond to the error bars along the $\mathrm{x}$ - and $\mathrm{y}$-axis respectively in the coming figures. Finally, a target corresponded to one pad and the pad thickness was measured prior and after testing to ascertain no substantial damage/wear.

(a)

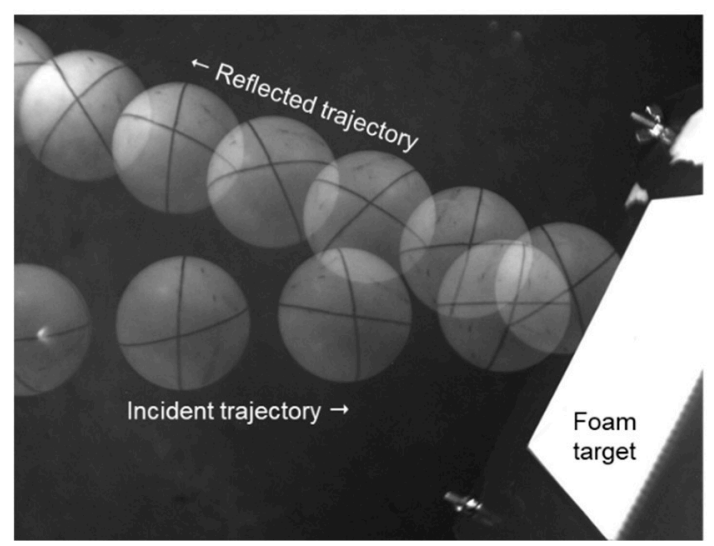

(b)

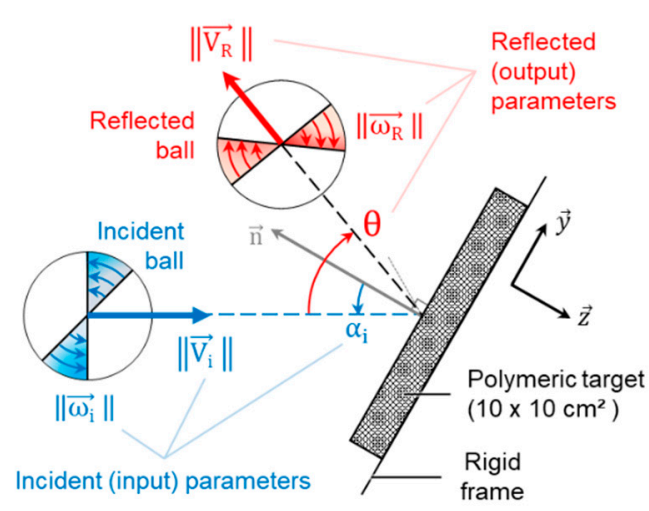

Figure 3. (a) Raw image of an impact test showing traces of the ball before and after contact. (b) Schematization of the kinematic parameters describing the motion of the table tennis ball prior (incident) and after (reflected) impact.

\subsection{Image Post-Processing and Metrics of Performance}

Post-processing software, property of Cornilleau@, was employed to determine the ball's complex trajectory from the obtained images. The non-orthogonal markers (Figure 2c) were recognized on the traces so that the 2D trajectory could be reconstructed [23,24]. Six kinematic parameters were finally monitored. They are displayed in Figure $3 \mathrm{~b}$. Three parameters were related to the incident trajectory:

- $\quad \alpha_{\mathrm{i}}$ is the incident angle, in degrees, calculated from the normal to the target surface $\vec{n}$.

- $\left\|\vec{V}_{\mathrm{i}}\right\|$ (also denoted $\mathrm{V}_{\mathrm{i}}$ in the following) corresponds to the magnitude, in meter per second $(\mathrm{m} / \mathrm{s})$, of the incident linear velocity. Considering the experimental setup and coordinate system defined in Figure $3 b, \vec{V}_{i}$ had components along the in-plane y- and z-axes,

- $\quad\left\|\vec{\omega}_{\mathrm{i}}\right\|$ (also denoted $\omega_{\mathrm{i}}$ in the following) corresponds to the magnitude, in revolution per second (rev/s), of the incident angular velocity (spin). Here, $\vec{\omega}_{\mathrm{i}}$ was collinear with the out of plane $x$-axes. Three parameters fully defined the reflected trajectory:

- $\theta$ is the deviation angle in degrees, calculated from the incident orientation. Clockwise angles were set positive.

- $\left\|\overrightarrow{\mathrm{V}}_{\mathrm{R}}\right\|$ corresponds to the magnitude, in meter per second $(\mathrm{m} / \mathrm{s})$, of the reflected linear velocity. $\vec{V}_{R}$ had components along the in-plane y-and z-axes,

- $\quad\left\|\vec{\omega}_{\mathrm{R}}\right\|$ corresponds to the magnitude, in revolution per second (rev/s), of the reflected angular velocity (spin). $\vec{\omega}_{R}$ was collinear with the out of plane x-axes,

Two assumptions accompanied the previous definitions. First, the ball was supposed to remain in the horizontal (xy) plane, suggesting that the effect of gravity was negligible. It was confirmed by verifying (using the circle fit functionality in the image processing software imageJ $\odot$ ), that the areas of the ball traces remained constant in one image. Indeed, if the camera were fixed on top of the set-up, the gravity would have contributed to out of plan motion and resulted in smaller traces of the ball on the image. Second, both the incident and reflected trajectories were assumed straight (at least between two consecutive traces), neglecting potential aerodynamics effects. 
With the trajectories fully defined, quantitative metrics were set in order to quantify the performance of the impacted polymeric layers. In the previous study focusing on normal impact with no spin [22], the sole linear coefficient of restitution was considered, as defined below:

$$
\mathrm{CR}_{\mathrm{L}}=\frac{\left\|\overrightarrow{\mathrm{V}}_{\mathrm{R}}\right\|}{\left\|\overrightarrow{\mathrm{V}}_{\mathrm{i}}\right\|},
$$

$\mathrm{CR}_{\mathrm{L}}$ is unitless, positive, smaller than 1 (when the target is fixed) and related to the speed performance. $\mathrm{CR}_{\mathrm{L}}$ remains a relevant metric and was used in this study. The broader spectrum of impact conditions explored suggested the use of two other metrics, namely the deviation angle $\theta$ and the (unitless) spin ratio SR defined as:

$$
\mathrm{SR}=\frac{\vec{\omega}_{\mathrm{R}}}{\vec{\omega}_{\mathrm{i}}}
$$

As mentioned above, both incident and reflected spin axes were collinear with the out of plane $x$-axis. Thus, the spin ratio SR is a real number with absolute value that can be greater than 1 . The spin ratio was also studied by Allen et al. [25] in the case of tennis balls. Thus, the aforementioned three parameters were related to the ball's trajectory and aimed at characterizing the performance of the impact between a given $\{$ Ball - Target $\}$ combination.

\section{Experimental Results}

Figure 4 presents, in a double entry array form, an overview of the impact conditions tested on the foam sample. Impact tests with varying negative (anticlockwise) angular velocities and incident angles are reported in the different rows and columns respectively. All the tests were performed at a constant linear velocity of $14 \mathrm{~m} / \mathrm{s}$ and ambient temperature. Three different spin $\left(0,-33\right.$ and $\left.{ }^{\circ} 66 \mathrm{rev} / \mathrm{s}\right)$ and three different angles $\left(0,30^{\circ}\right.$ and $\left.60^{\circ}\right)$ were considered. The top left image corresponds to a normal impact with no spin that has been studied and documented in the previous study [22]. Under the new tested loading conditions, incident and reflected trajectories were easily distinguished.

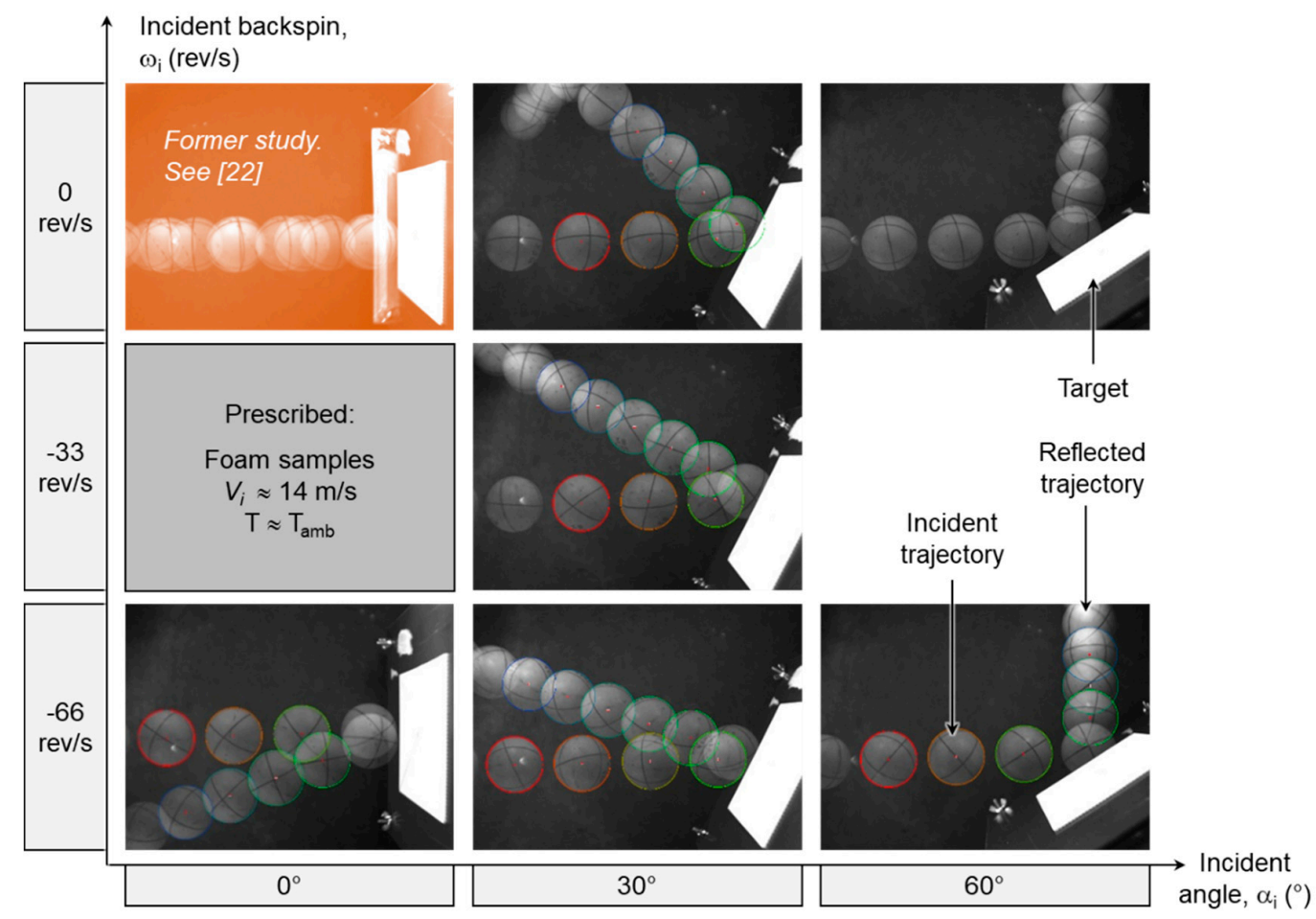

Figure 4. Raw images of the various impact tests performed on the foam sample. 
Studying the effect of the incident spin for a fixed angle, or the effect of the incident angle for a fixed spin was obtained by focusing on one row or column respectively. For instance, the effect of the incident angle when the ball was thrown at $14 \mathrm{~m} / \mathrm{s}$ with a backspin of $66 \mathrm{rev} / \mathrm{s}$ is presented in Figure 5 . Figure 5a displays the raw images of the impact performed on the foam + compact (row 1 ) and foam (row 2) samples. At first sight, noticeable differences in the reflected trajectories was observed between the two samples for a given $\left\{\omega_{\mathrm{i}} ; \alpha_{\mathrm{i}}\right\}$.

(a)
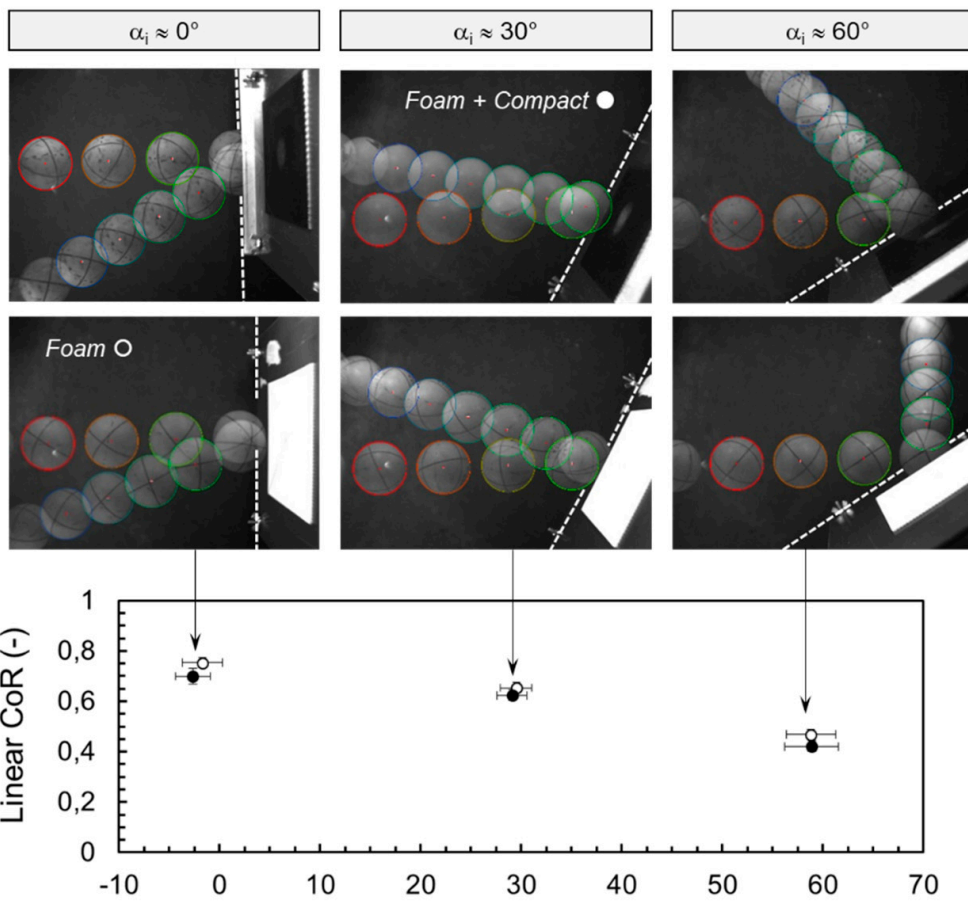

(b)
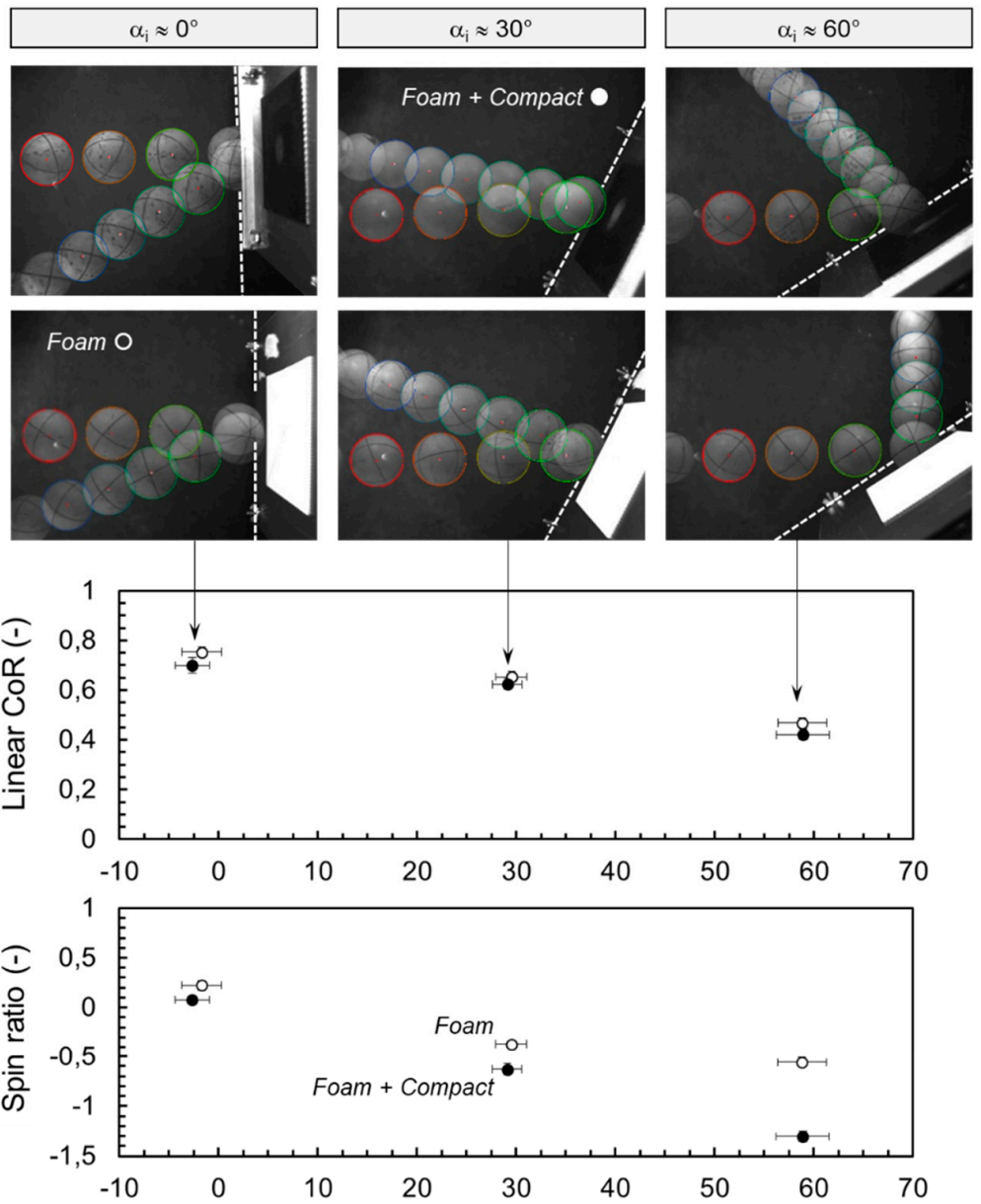

(c)

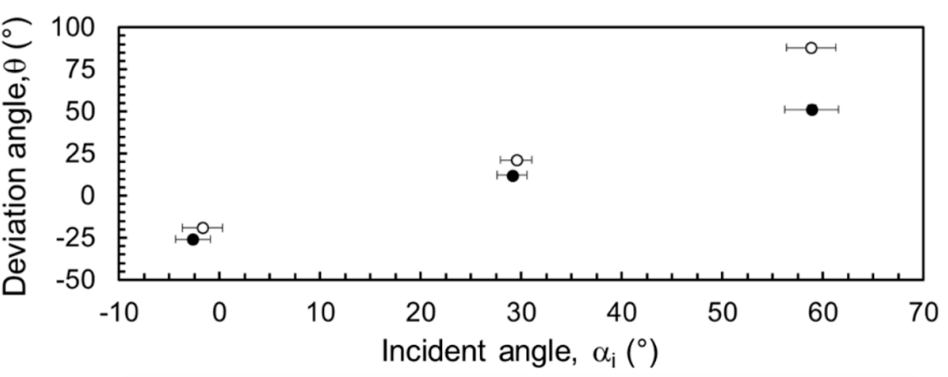

Figure 5. (a) Raw images of impact tests performed on the foam + compact and foam samples with varying incident angle $\left(\left\|\vec{V}_{\mathrm{i}}\right\|=14 \mathrm{~m} / \mathrm{s}, \mathrm{T}_{\mathrm{amb}}\right)$. (b) Linear coefficient of Restitution as a function of the incident angle. (c) Spin ratio as a function of the incident angle. (d) Deviation angle as a function of the incident angle.

Further quantitative analysis is presented in Figure $5 b-d$ where the linear coefficient of restitution, the spin ratio and the deviation angle are respectively plotted as a function of the incident angle. Increasing the incident angle led to a decrease in the linear coefficient of restitution, the values for the foam + compact being slightly smaller than those measured on the foam alone. 
For the two other metrics, a change in the sign was observed between the normal tests $\left(\alpha_{i}=0\right)$ and the tilted ones $\left(\alpha_{i} \neq 0\right)$. For the normal tests, the spin did not change direction and the ball bounced back with a negative deviation angle. For tilted tests, the spin sign did change leading to negative spin ratio and positive deviation angles.

Focusing on the magnitudes, here again a clear distinction could be made between the normal and tilted tests. For normal impacts, the foam + compact sample led to a larger deviation angle and a smaller spin ratio than the foam specimen. The opposite conclusions prevailed when the incident angle was set equal to 30 or $60^{\circ}$. Finally, the greater the incidence, the larger the difference between the two targets.

A companion study where the incident angle was prescribed to $30^{\circ}$ and the incident backspin varied, is presented in Figure 6.
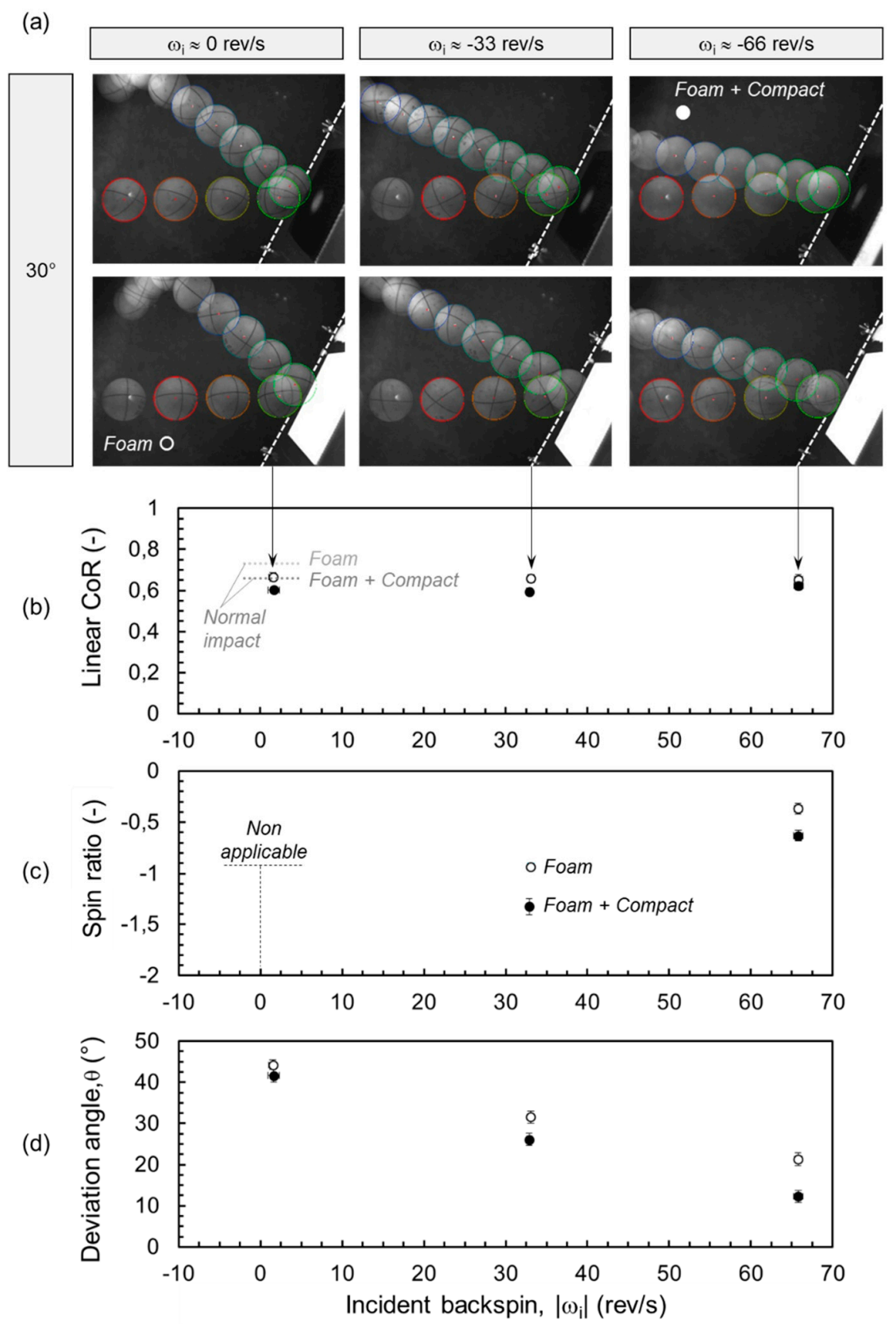

Figure 6. (a) Raw images of impact tests performed on the foam + compact and foam samples with varying angular velocity $\left(\left\|\vec{V}_{\mathrm{i}}\right\|=14 \mathrm{~m} / \mathrm{s}, \mathrm{T}_{\mathrm{amb}}\right)$. (b) Linear coefficient of Restitution as a function of the incident backspin. (c) Spin ratio as a function of the incident backspin. (d) Deviation angle as a function of the incident backspin. 
Figure 6a displays the raw images of the impact performed on the foam + compact (row 1 ) and foam (row 2) samples and Figure 6b-d display respectively the evolution of the linear coefficient of restitution, the spin ratio and the deviation angle as a function of the incident backspin. The linear coefficient of restitution is seen to remain almost constant, with the foam values remaining slightly larger than the foam + compact ones. For the sake of comparisons, the values gathered in [22] for normal impact with no spin were superimposed, evidencing that a deviation from the normal incidence led to a decrease in the $\mathrm{CR}_{\mathrm{L}}$ of about 10 percent. Regarding the spin ratio reported in Figure $6 \mathrm{~b}$, negative values were found and its magnitudes decreased as the backspin increased, with larger values being found for the compact + foam sample. Finally, the deviation angle decreased with increasing backspin. The foam sample exhibited the larger angles compared to the foam + compact sample and the gap increased with increasing backspin.

In summary, distinct discrepancies were experimentally found between the foam and foam + compact samples response to impact. Whatever the impact condition tested, the linear coefficient of restitution of the foam + compact was smaller than the one of the foam alone. Also, normal impact aside, impact tests performed on the foam + compact target resulted in a larger spin ratio and a smaller deviation angles.

\section{Modeling Ball/Pad Impact}

As outlined in the introduction, the objective of this section consisted in challenging, with this new set of data, the modelling strategy that had been implemented in [22] to simulate normal impact with no spin. Thus, only minor adjustments were applied to the finite element model. For instance, the constitutive behavior of the constituents remained unchanged. The major modeling assumptions are recalled in the following two first subsections.

\subsection{Finite Element Model Description}

Explicit 3D finite elements simulations using the commercial software ABAQUS@ [26] were pursued to model the impact experiments. With this modeling strategy, the geometry/architecture, the materials intrinsic behavior and the contact properties of the mechanical problem were considered.

The 3D model is presented in Figure 7a. The ball comprises 5058 S4R shell elements with five integration points through the thickness and the polymeric layers is made of 221,598 C3D8R elements: 100,314 elements for the foam layer, 26,028 for the periodic array of perfectly cylindrical pimples and 95,256 elements for the dense top layer of the compact. This distinction has been made since these three layers were modelled as different instances that were bonded together when needed, using the tie function between the shared surfaces as evidenced in the inset of Figure $7 \mathrm{~b}$.

(a)

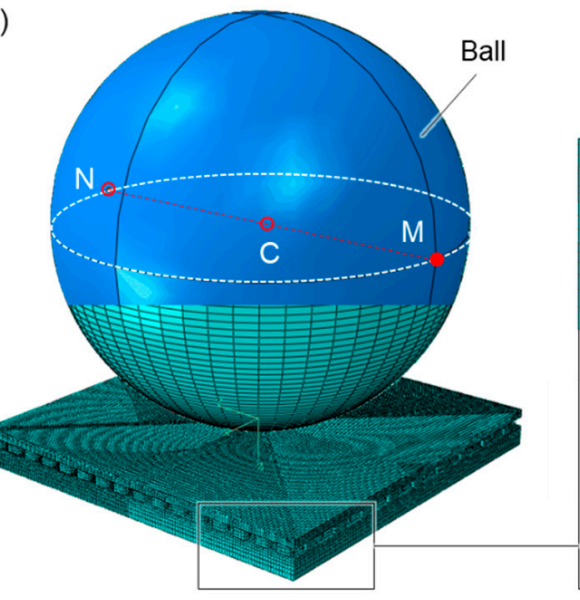

(b)

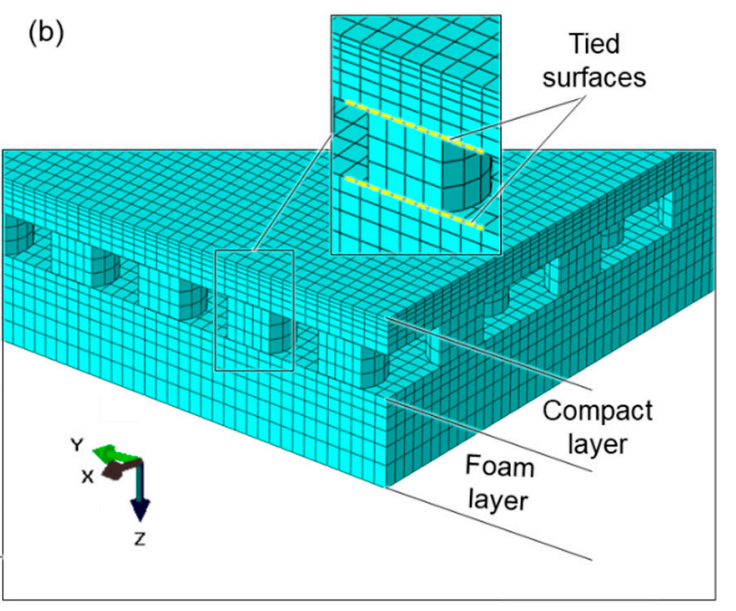

Figure 7. Finite element modeling (FEM). (a) 3D model and associated meshes. (b) Details on the foam + compact modeling. 
The nodes of the foam bottom free surface were fixed to replicate its gluing onto the wooden rigid frame. At time $t=0$ the ball was positioned $0.3 \mathrm{~mm}$ away from the top surface of the target. In order to ensure its rigid motion, the initial velocity was assigned to every node $\mathrm{M}$ of the ball and was calculated using the following transport equation:

$$
\overrightarrow{\mathrm{V}}_{\mathrm{M}}=\overrightarrow{\mathrm{V}}_{\mathrm{C}}+\overrightarrow{\mathrm{w}}_{\mathrm{C}} \wedge \overrightarrow{\mathrm{CM}}
$$

with $\vec{V}_{C}$ and $\vec{\omega}_{C}$ the linear and angular velocities of the ball center $C$. The velocity was applied for a short duration $\left(10^{-6} \mathrm{~s}\right)$ and the ball was then let free to move (the drag force and gravity were not implemented). Also, constant internal pressure equal to the atmospheric pressure was prescribed as justified elsewhere [8].

The contact between the two bodies was defined rough if not otherwise specified. More precisely, the rough condition implies an infinite coefficient of friction (total adhesion), suggesting that all relative sliding motion between two contacting surfaces is prevented [27]. It is worth mentioning that this hypothesis was used in [22] to successfully predict the linear coefficient of restitution of the various polymeric targets normally impacted. This hypothesis is strongly challenged and discussed with this new set of data.

\subsection{Intrinsic Behavior of the Constituents}

A rate-dependent dissipative elastic description was used to describe the ball parent glassy material since it did not overcome its elastic limit under the large set of impact tested. The foam and the compact were defined as rate-dependent dissipative hyperelastic materials. The same constitutive equation was used for both layers as justified in [22].

Dynamic mechanical analysis (DMA) tests and time-temperature superposition principles were used to characterize the dissipative rate dependence elastic behavior of the different materials. Additional quasi-static compression test was performed on the foam system to characterize its large non-linear deformation behavior. An overview of the DMA response in the tensile mode of the ball parent material and the foam are displayed in Figure 8a,b respectively, and the large strain compression response of the foam is presented in Figure 8c. The reader should refer to [22] for further details on the methods and testing conditions.

(a)

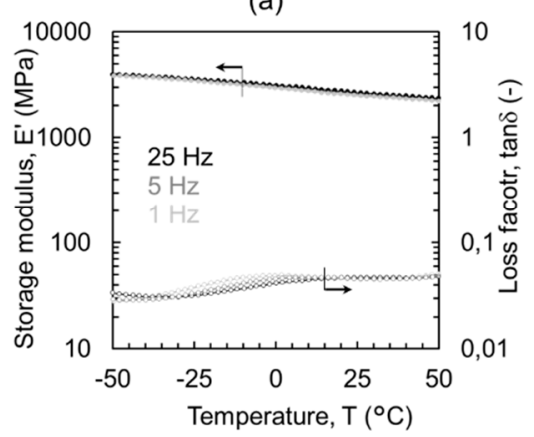

(b)

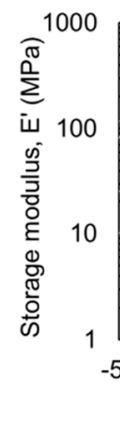

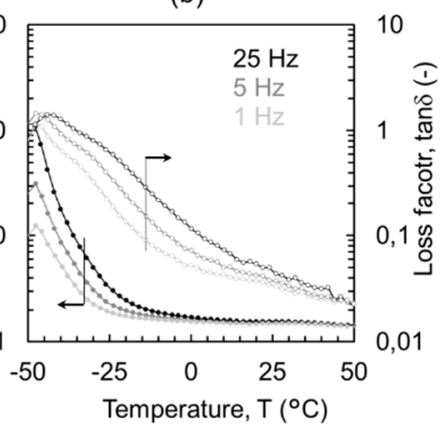

(c)

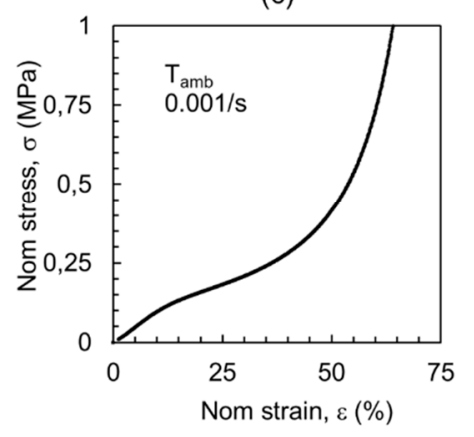

Figure 8. (a) Isochronal dynamic mechanical analysis (DMA) profiles of the ball parent material in the $[-50 ; 50]{ }^{\circ} \mathrm{C}$ temperature range. (b) Isochronal DMA profiles of the foam material in the $[-50 ; 50]{ }^{\circ} \mathrm{C}$ temperature range. (c) Compression stress strain response of the foam parent material. N.B. This figure is a reproduction of Figure 3 in [22].

In ABAQUS $\odot$, Prony series fitting the experimentally based time-dependent shear modulus of visco-elastic materials $G(t)$ were implemented. Nine discrete Maxwell elements were identified for the ball and the foam (and compact). Constant Poisson ratio were allocated to the polymeric constituents to control the volumetric behavior. Finally, table data of the experimental strain-stress response was used to fit the isotropic Ogden strain energy potential for the long-time hyperelastic 
behavior of the polymeric coatings layers [27]. It is worth noting that the Mullins and Payne effects of the elastomeric layers were neglected and their potential effects minimized experimentally (by testing and characterizing fatigued samples on the one hand and controlling the recovering time between consecutive impact tests) [28].

Also, the foam was modeled as a continuum media whereas the exact architecture of the compact layer was modeled. Consequently, their density and Poisson ratio differ. Almost incompressible Poisson ratio $\left({ }^{C} v=0.49\right)$ and density ${ }^{C} \rho=1000 \mathrm{~kg} / \mathrm{m}^{3}$ were assigned to the compact parent material whereas effective values of ${ }^{\mathrm{F}} \rho=490 \mathrm{~kg} / \mathrm{m}^{3}$ and ${ }^{\mathrm{F}} v=0.41$ (determined using video-extensometry) were measured and implemented for the foam.

\subsection{Experiments/Simulations Comparisons}

While the ball's simulated incident trajectory was an input, its reflected trajectory needed to be determined in order to compute the aforementioned metrics. Thus, the position of the center of the ball led to the determination of deviation angle and the linear coefficient of restitution and was calculated from the coordinates at any time increment of two nodes, denoted $\mathrm{M}$ and $\mathrm{N}$ in Figure 7a, located away from the contact region. The center was half way between $\mathrm{M}$ and $\mathrm{N}$, assuming that these two nodes corresponded at any time to the ball's diameter.

With this assumption, the rotation angle $\beta$ of the ball with respect to the out of plane $x$-axis was determined as follows:

$$
\beta=\operatorname{atan}\left(\frac{z_{N}-z_{M}}{y_{N}-y_{M}}\right)
$$

with $\left\{\mathrm{y}_{\mathrm{N}}, \mathrm{z}_{\mathrm{N}}\right\}$ and $\left\{\mathrm{y}_{\mathrm{M}}, \mathrm{z}_{\mathrm{M}}\right\}$ the in-plane $\mathrm{y}$ - and z-coordinates of the $\mathrm{N}$ and $\mathrm{M}$ points respectively. The associated angular velocity (spin) was then determined from two consecutive time increments:

$$
\omega=\frac{\Delta \beta}{\Delta t}
$$

with $\Delta \beta$ and $\Delta \mathrm{t}$ the angle and time increments, respectively.

Figure 9 presents the simulated vertical displacement mapping for a ball impacting the foam + compact target at a $60^{\circ}$ angle, $14 \mathrm{~m} / \mathrm{s}$ and $-66 \mathrm{rev} / \mathrm{s}$. Four screenshots are displayed corresponding to the initial time $\left(t_{0}\right)$, first contact $\left(t_{1}\right)$, contact at maximum depth $\left(t_{2}\right)$ and end time $\left(t_{\text {end }}\right)$. A close up view of the through-thickness deformation in the contact region at time $t_{2}$ is added to evidence the complex deformation pattern of the target layers. It is worth noting that no buckling of the ball occurred in this case and that the pimples were significantly sheared.

The positions of the $\mathrm{N}$ and $\mathrm{M}$ equatorial points, and a fictitious line connecting them are also displayed. As discussed above, their time positions were necessary for the metrics calculations. Also, the trajectories of the $\mathrm{M}, \mathrm{N}$ and calculated $\mathrm{C}$ points are plotted in Figure 10. The positions for the times selected in Figure 9 are presented and the spin inversion is clearly evidenced.

In the previous study focusing on the normal impact with no spin, the linear coefficient of restitution was the sole relevant metric monitored. Also, finite element simulations with rough contact conditions resulted in fair predictions of the experimental data compared to simulations with frictionless ones. A similar study had been conducted in this work for the foam sample impacted under various incidences and spins. The results are presented in Figure 11 where the experimental and simulated linear coefficient of restitutions are compared.

Figure 11a focuses on impacts with fixed backspin $(-66 \mathrm{rev} / \mathrm{s})$ and varying incidence and Figure $11 \mathrm{~b}$ presents the results for impacts at constant incidence $\left(30^{\circ}\right)$ and varying backspin. Fair predictions using rough contact conditions were obtained whereas frictionless simulations clearly over predicted the experimental results. With this broad set of impact conditions, the numerical predictions capability using rough contact conditions could be further challenged when tracking the two additional metrics that had been defined. 


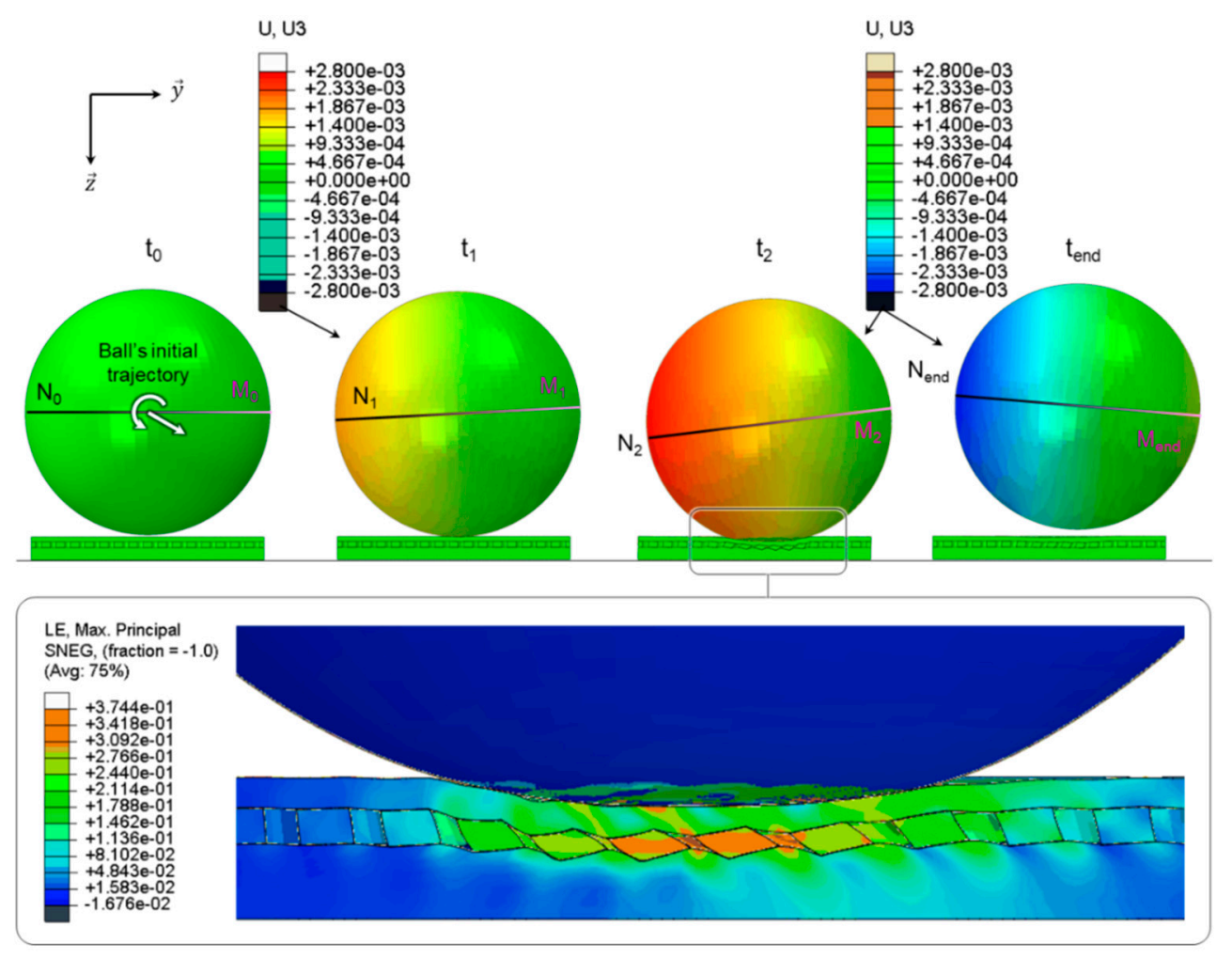

Figure 9. Simulated vertical displacement mapping of a ball impacting the foam + compact target at 4 distinct times $\left(\alpha_{i}=60^{\circ}\right.$ angle, $V_{i}=14 \mathrm{~m} / \mathrm{s}$ and $\left.\omega_{i}=-66 \mathrm{rev} / \mathrm{s}\right)$. The inset figure focuses on the through-thickness deformation profile at maximum depth during contact.

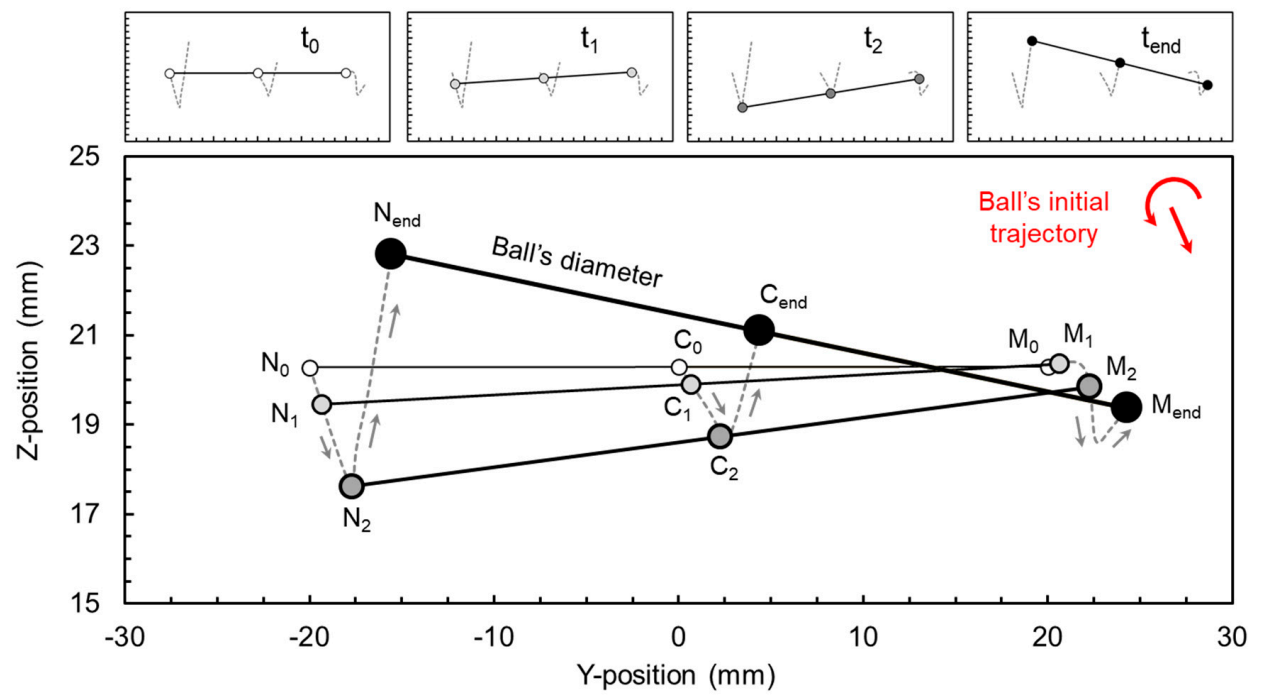

Figure 10. In-plane trajectories of the $M, N$ and calculated $C$ points of the ball. $\left(\alpha_{i}=60^{\circ}\right.$ angle, $\mathrm{V}_{\mathrm{i}}=14 \mathrm{~m} / \mathrm{s}$ and $\left.\omega_{\mathrm{i}}=-66 \mathrm{rev} / \mathrm{s}\right)$. N.B. different scales were used for $\mathrm{y}$ and $\mathrm{z}$.

The experiment/simulation comparisons are presented in Figure 12 for impact tests at constant velocities $\left(\mathrm{V}_{\mathrm{i}}=14 \mathrm{~m} / \mathrm{s}\right.$ and $\left.\omega_{\mathrm{i}}=-66 \mathrm{rev} / \mathrm{s}\right)$ and varying incidence, performed on the foam (Figure 12a) and foam + compact (Figure 12b) targets. For both the foam and the foam + compact targets, the magnitude of the linear coefficient of restitution and its decrease with increasing the incident angle were fairly predicted (top figures in Figure 12). 
(a)

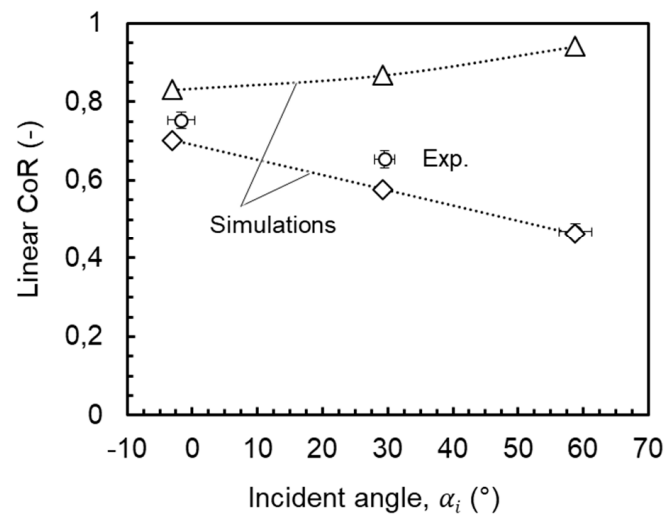

(b)

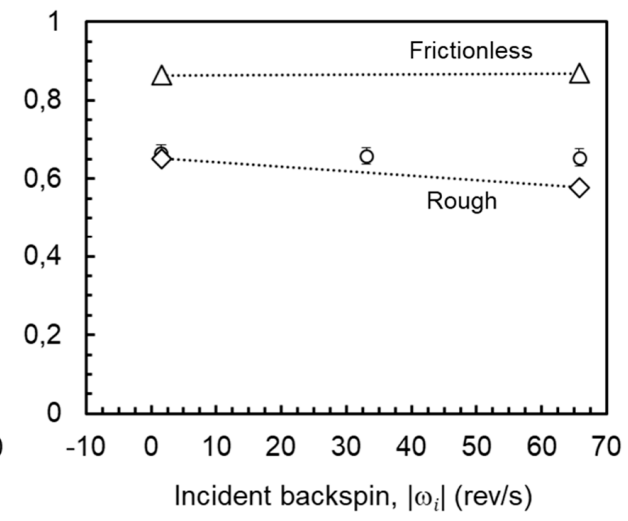

Figure 11. Effect of the ball/foam contact properties on the predicted linear coefficient of restitutions. (a) Foam impacted at varying incidence and constant velocities $\left(\mathrm{V}_{\mathrm{i}}=14 \mathrm{~m} / \mathrm{s}\right.$ and $\left.\omega_{\mathrm{i}}=-66 \mathrm{rev} / \mathrm{s}\right)$. (b) Foam impacted at varying incident backspin and constant angle $\left(\alpha_{i}=30^{\circ}\right)$ and linear velocity $\left(V_{i}=14 \mathrm{~m} / \mathrm{s}\right)$.

(a) Foam
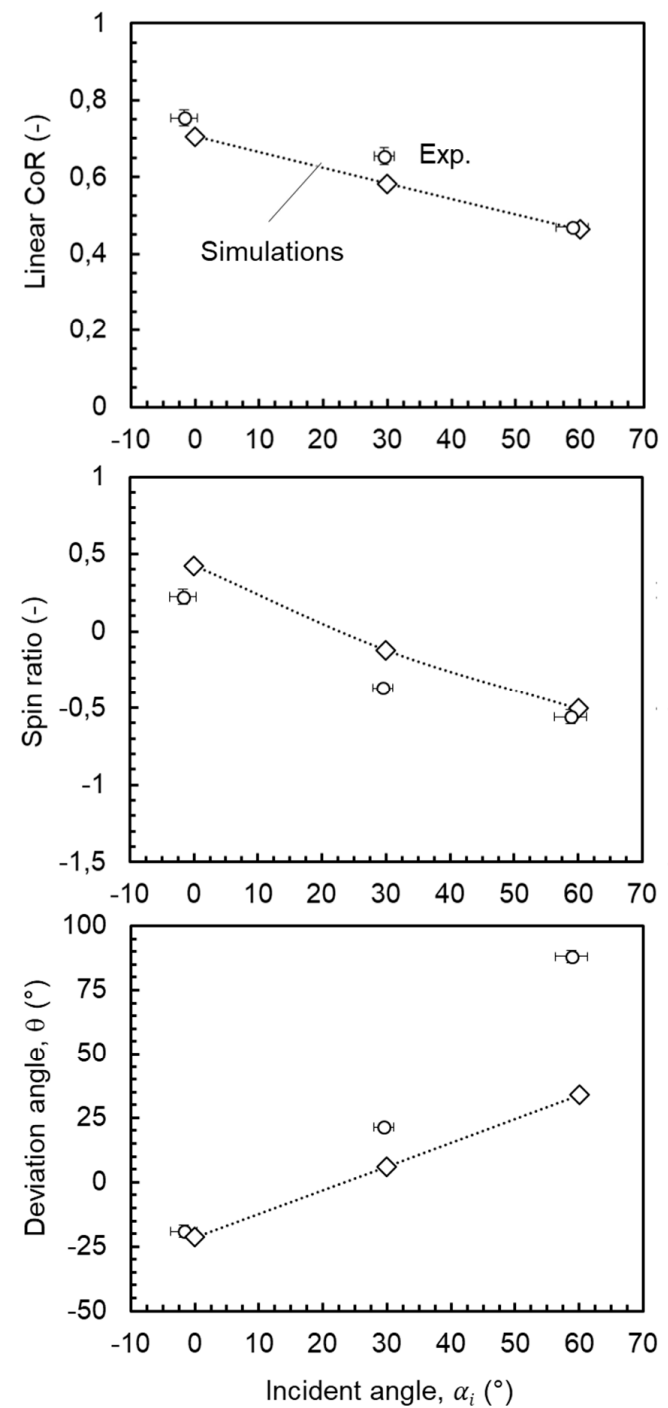

(b) Foam + Compact
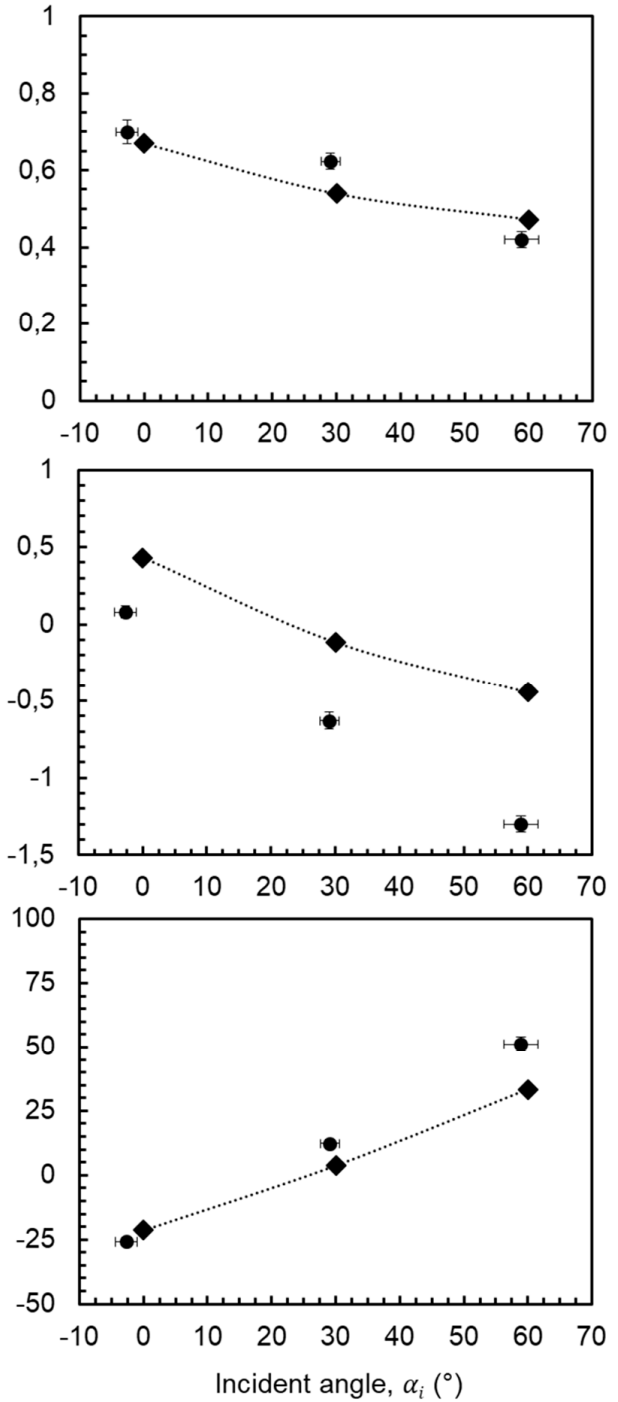

Figure 12. Model/experiments confrontations of the three metrics of performance as a function of the incident angle for impact $\left(\mathrm{V}_{\mathrm{i}}=14 \mathrm{~m} / \mathrm{s}\right.$ and $\left.\omega_{\mathrm{i}}=-66 \mathrm{rev} / \mathrm{s}\right)$. (a) foam sample. (b) foam + compact sample. 
Furthermore, the qualitative evolutions of the two other metrics, that were an inversion and then an increase of the absolute value of the spin ratio and an increase of the deviation angle, were both captured. Yet, the experimental and simulated magnitudes strongly differed. More precisely, limited differences could be observed between the simulated metrics of the two targets whereas large experimental discrepancies were already reported: normal impact aside, impacts on the foam + compact sample resulted in larger spin ratio magnitudes and smaller deviation angles $\theta$.

These differences suggested that the ball/foam and ball/compact contacts were different. At first glance, the ball/foam friction coefficient seemed smaller than the compact/foam one. Nevertheless, the potential difference between the deforming contact geometries could not be ignored. Thus, complementary studies of the contact shape with account for the refined and potentially different contact properties between the foam and the compact layers need to be pursued. Numerically, parametric study with varying coefficient of friction (CoF) can be performed. Experimentally, its measurement can be obtained as reported in $[29,30]$ for instance. These refinements are further justified since the reversible buckling of the ball (leading to very different contact areas) only occurred for certain experimental conditions tested. These investigations are currently pursued. Also, in the present study, the contact forces (normal and tangent) were not considered. Future works will consider the implementation of a force plate to measure the impact of normal and tangent forces and compare with the ones predicted by the finite element analysis, as in $[18,19]$. This will probably help in tuning the friction coefficient between the ball and the covering.

\section{Conclusions}

The impact of a table tennis ball onto part or the complete polymeric racket layers had been investigated for various incident angles and spins. Three metrics of performance, namely the linear coefficient of restitution $\mathrm{CR}_{\mathrm{L}}$, the spin ratio $\mathrm{SR}$ and the deviation angles $\theta$ were monitored evidencing strong differences between the foam and foam + compact layers. In short, normal spin aside, the ball impacting the foam pad exhibited smaller reflected spin and larger reflected angle than the one impacting the foam + compact.

The numerical prediction of the impact had been attempted using a previously implemented 3D finite element model that happened to successfully predict the linear coefficient of restitution of the same target under normal impact with no spin. The rate dependent dissipative constitutive equations of the ball and polymeric layers were determined based on DMA and the large strain hyperelastic response was obtained from quasi-static compression tests.

While the linear coefficient of restitution was fairly predicted, the two other metrics failed to be successfully simulated, revealing the key role of the contact condition. The simplistic rough contact condition needs to be refined.

However, the numerical tool comprises all the required elements to study the impact of a table tennis ball on the racket polymeric layers. Ongoing work focuses on a better account for the contact, and detailed analysis of the simulated contact region is attempted in order to get a better sense of the role of the contact geometry. In addition, the in-depth study of the energy balance (strain, kinetic, viscous, friction components) is currently being pursued to identify the key mechanisms driving the overall performance of the polymeric layers impacted by a table tennis ball.

Author Contributions: Conceptualization, R.G.R., L.M., S.M. and N.H.; methodology, R.G.R., L.M., S.M. and N.H.; software, R.G.R. and L.M.; validation, R.G.R., L.M., S.M. and N.H.; formal analysis, R.G.R. and L.M.; investigation, R.G.R. and L.M.; resources, R.G.R., L.M., S.M. and N.H.; data curation, R.G.R., L.M.; writing一original draft preparation, R.G.R., L.M.; writing—review and editing, R.G.R., L.M..; visualization, R.G.R.; supervision, R.G.R., L.M.; project administration, N.H.; funding acquisition, N.H.

Funding: This research received no external funding.

Acknowledgments: The long-term collaboration between INSA Lyon and the French company Cornilleau@ consists of incremental student projects over the years. Consequently, the numerous students who have been involved over the years deserved to be acknowledged for their implications and efforts. Here they are, chronologically listed: T. Huin, A. Drillon, C. Bonnard, Z. Hui, L. Marin Curtoud, D. Quanshangze. Special thanks 
also go to H. Lourenco for his help during the experimental campaigns performed at Cornilleau in the early years of this collaboration.

Conflicts of Interest: The authors declare no conflict of interest.

\section{References}

1. Kamijima, K.; Ushiyama, Y.; Yasaka, T.; Ooba, M. Effect of different playing surfaces of the table on ball bounces in table tennis. Int. J. Table Tennis Sci. 2013, 1, 11-14.

2. Nagurka, M.; Huang, S. A mass-spring-damper model of a bouncing ball. In Proceedings of the 2004 American Control Conference, Boston, MA, USA, 30 June-2 July 2004.

3. Nakashima, A.; Ito, D.; Hayakawa, Y. An online trajectory planning of struck ball with spin by table tennis robot. In Proceedings of the 2014 IEEE/ ASME International Conference Advanced Intelligent Mechatronics (AIM), Besançon, France, 8-11 July 2014.

4. Nakashima, A.; Okamoto, T.; Hayakawa, Y. An online estimation of rotational velocity of flying ball via aerodynamics. IFAC Proc. Vol. 2014, 47, 7176-7181. [CrossRef]

5. Nonomura, J.; Nakashima, A.; Hayakawa, Y. Analysis of effects of rebounds and aerodynamics for trajectory of table tennis ball. In Proceedings of the SICE Annual Conference 2010, Taipei, Taiwan, 18-21 August 2010.

6. An, C.-C.; Hsu, H.Y.; Sun, Y.T.; Ke, L.D.; Hsu, T.G.; Ting, C.C. Developing an audio analyzer for instantaneous stroke position identification on table tennis racket to assist technical training. Measurement 2018, 115, 73-79. [CrossRef]

7. Widenhorn, R. The physics of juggling a spinning ping-pong ball. Am. J. Phys. 2016, 84, 936-942. [CrossRef]

8. Zhang, X.; Tao, Z.; Zhang, Q. Dynamic behaviors of visco-elastic thin-walled spherical shells impact onto a rigid plate. Lat. Am. J. Solids Struct. 2014, 11, 2607-2623. [CrossRef]

9. Dong, X.; Gao, Z.; Yu, T. Dynamic crushing of thin-walled spheres: An experimental study. Int. J. Impact Eng. 2008, 35, 717-726. [CrossRef]

10. Pauchard, L.; Rica, S. Contact and compression of elastic spherical shells: The physics of a 'ping-pong' ball. Philos. Mag. B 1998, 78, 225-233. [CrossRef]

11. Ruan, H.; Gao, Z.; Yu, T. Crushing of thin-walled spheres and sphere arrays. Int. J. Mech. Sci. 2006, 48, 117-133. [CrossRef]

12. Shorter, R.; Smith, J.; Coveney, V.; Busfield, J. Axial compression of hollow elastic spheres. J. Mech. Mater. Struct. 2010, 5, 693-705. [CrossRef]

13. Cho, H.; Rinaldi, R.G.; Boyce, M.C. Constitutive modeling of the rate-dependent resilient and dissipative large deformation behavior of a segmented copolymer polyurea. Soft Matter 2013, 9, 6319-6330. [CrossRef]

14. Rinaldi, R.G.; Hsieh, A.; Boyce, M. Tunable microstructures and mechanical deformation in transparent poly (urethane urea) s. J. Polym. Sci. Part B Polym. Phys. 2011, 49, 123-135. [CrossRef]

15. Tiefenbacher, K.; Durey, A. The impact of the Table Tennis Ball on the Racket (Backside Coverings). Int. J. Table Tennis Sci. 1994, 2, 1-14.

16. Tiefenbacher, K.; Seydel, R.; Durey, A. Analysis of the influence of special equipment materials on decisive strokes. Int. J. Table Tennis Sci. 1996, 2, 51-60.

17. Cross, R. Grip-slip behaviour of a boucing ball. Am. J. Phys. 2002, 70, 1093-1102. [CrossRef]

18. Goodwill, S.R.; Kirk, R.; Haake, S.J. Experimental and finite element analysis of a tennis ball impact on a rigid surface. Sports Eng. 2005, 8, 145-158. [CrossRef]

19. Allen, T.; Goodwill, S.; Haake, S. Experimental validation of a tennis ball finite-element model. In Tennis Science and Technology 3; Miller, S., Capel-Davies, J., Eds.; International Tennis Federation: London, UK, 2007; pp. 23-30.

20. Allen, T.; Goodwill, S.; Haake, S. Experimental validation of a tennis ball finite-element model for different temperatures (P22). In The Engineering of Sport 7; Springer: Paris, France, 2009.

21. Allen, T.; Haake, S.; Goodwill, S. Comparison of a finite element model of a tennis racket to experimental data. Sports Eng. 2009, 12, 87-98. [CrossRef]

22. Rinaldi, R.G.; Manin, L.; Bonnard, C.; Drillon, A.; Lourenco, H.; Havard, N. Non linearity of the ball/rubber impact in table tennis: Experiments and modeling. Procedia Eng. 2016, 147, 348-353. [CrossRef]

23. Carre, M.J.; Haake, S.; Baker, S.W.; Newell, A. The analysis of cricket ball impacts using digital stroboscopic photography. In The Engineering of Sport_Design and Development; Blackwell: Oxford, UK, 1998; pp. 379-386. 
24. Theobalt, C.; Albrecht, I.; Haber, J.; Magnor, M.; Seidel, H.P. Pitching a baseball: tracking high-speed motion with multi-exposure images. ACM Trans. Graph. 2004, 23, 540-547. [CrossRef]

25. Allen, T.; Ibbitson, J.; Haake, S. Spin generation during an oblique impact of a compliant ball on a non-compliant surface. Proc. Inst. Mech. Eng. Part P J. Sports Eng. Technol. 2012, 226, 86-95. [CrossRef]

26. ABAQUS, Analysis User's Manual, Version 6.12; Karlsson \& Sorensen, Inc.: Pawtucket, RI, USA, 2012.

27. ABAQUS: Theory manual; Hibbit, Karlsson \& Sorensen, Inc.: Pawtucket, RI, USA, 1997.

28. Ward, I.M.; Sweeney, J. Mechanical Properties of Solid Polymers; John Wiley \& Sons: Hoboken, NJ, USA, 2012.

29. Varenberg, M.; Varenberg, A. Table tennis rubber: Tribological characterization. Tribol. Lett. 2012, 47, 51-56. [CrossRef]

30. Varenberg, M.; Varenberg, A. Table Tennis: Preliminary Displacement in Pimples-Out Rubber. Tribol. Lett. 2014, 53, 101-105. [CrossRef]

(C) 2019 by the authors. Licensee MDPI, Basel, Switzerland. This article is an open access article distributed under the terms and conditions of the Creative Commons Attribution (CC BY) license (http://creativecommons.org/licenses/by/4.0/). 